\title{
Throughput of Inhibit Sense Multiple Access with Propagation Delays
}

\author{
Jean-Paul M.G. Linnartz, Member, IEEE, Geert A. Awater, Student Member, IEEE and Robert-Jan Venema
}

\begin{abstract}
A large number of terminals transmitting data packets over a common radio channel to a central base station is studied. In inhibit sense multiple access (ISMA), the base station broadcasts a busy signal when an incoming packet is being received, to inhibit other terminals from colliding transmissions. This busy signal arrives at each terminal with a propagation delay, proportional to the distance between the base station and the terminal. This leads to unfairness in the probability of successfully transmitting a data packet, since nearby terminals have more up-to-date information on the actual channel status than remote terminals. This unfairness is additional to the advantage that nearby terminals have because of the capture effect, which is also considered here. The paper applies nonstationary Poisson processes to describe the random arrival of data packets at the central receiver. It is shown that the probability of a successful attempt to transmit a packet decreases, approximately linearly with the distance between the transmitter and the central receiver. The total throughput is also assessed and is found that the assumption of a fixed propagation delay adopted in CSMA studies by others gives too optimistic results for ISMA. Moreover, our analysis suggests a subtle change of the fixed delay approximation that enhances its accuracy, withou adding complexity. The effect of propagation delays in random access radio networks are of increasing importance, since newly developed systems are designed to transmit with increasingly high bit rates.
\end{abstract}

\section{INTRODUCTION}

$\mathbf{P}$ ACKET switching in wireless (radio) channels relies on appropriate access protocols to organize randomly occurring attempts by terminals, to transmit a data packet to a central station (e.g., a host computer). The ALOHA system [1] is one of the earliest examples of packet-switching of data over radio channels, to provide a means of communication between a number of geographically distributed terminals and a central (host) computer. In the ALOHA protocol, terminals transmit packets over a common radio channel without any mutual control or regulation. If two or more terminals happen to transmit simultaneously, a packet 'collision' occurs. This mutual interference results in loss of packets, which have to be retransmitted. To reduce the adverse effects of collisions, a number of alternative protocols have been proposed. In carrier sense multiple access (CSMA), a terminal with a packet to

Paper approved by Leonard J. Cimini, Jr., the Editor for Mobile Communications of the IEEE Communications Society. Manuscript received March 28, 1991; revised July 17, 1992

The J.-P. M. G. Linnartz is with the Telecommunications and TrafficControl Systems Group, Delft University of Technology, The Netherlands, on leave from the University of California, Berkeley

G. A. Awater and R.J. Venems are with the Telecommunications and Traffic-Control Systems Group, Delft University of Technology, The Netherlands.

IEEE Log Number 9215051 be transmitted, first senses the channel for active carriers from other terminals [2]. Only if the channel is idle, a new data packet is allowed to be transmitted. In mobile radio systems, CSMA has the drawback that collisions can occur if a terminal is not aware of an ongoing transmission by another remote terminal. This issue is discussed as the hidden terminal problem in [3]. In the absence of propagation delays, this problem is avoided in ISMA, where the central station continuously broadcasts the status of the inbound channel, being either IDLE or BuSY, to all terminals. In [4], where the benefits of a channel status feedback on system throughput are established through an analytical approach, on-off keying of a carrier that is broadcast by the central station is proposed to implement this signaling channel

This paper addresses non-persistent ISMA: transmission of the packet only occurs if the terminal is granted permission to transmit, i.e. if an idle signal is received on the signalling channel. Any transmission attempt arriving when the terminal receives a busy signal does not lead to a transmission and is therefore not successful. Such an 'inhibited' packet is rescheduled for a later attempt. Since the feedback signal is received only after a certain delay, remote terminals have a retarded view of the channel status. Therefore, packet collisions can still occur. Kleinrock and Tobagi [2] dealt with a propagation delay that is identical for all participating terminals, irrespective of their distance to the central station. The effect of delays increasing, proportional with the propagation distance over a wired LAN network, was investigated by Molle, Sohraby and Venetsanopoulos in [5]. The present paper addresses radio networks and deals with delays that consist of a fixed part called the 'processing delay,' and a distance-dependent part called the 'propagation delay.' The processing delay is caused by the necessary operations performed in the central station, for instance the recognition of an incoming packet signal. The transmitter power-up time in the mobile terminal may also largely contribute to the fixed (processing) delay [6]. Propagation delays may be negligible, compared to the processing delays in a typical cellular mobile data network employing a low bit rate, such as $1200 \mathrm{bit} / \mathrm{s}$. Presently, however, new mobile information systems are designed for increasingly high data rates. For instance, in a high-capacity mobile data net with a service area of $30 \mathrm{~km}$, packets of 200 bits and a bit rate of $200 \mathrm{kbit} / \mathrm{s}$, with a packet duration of 1 ms, the maximum round-trip delay is $20 \%$ of the duration of a packet. Propagation delays of this order of magnitude may seriously affect the system performance. This observation has motivated the authors to investigate the issue. The exemplary 
parameters above will, in fact, be used in the computational examples further on.

Propagation delays, unlike processing delays, lead to unfairness in the probability to perform a successful packet transmission. The near-far effect caused by the fact that distant terminals have more retarded information of the receiver status than nearby terminals, is additional to the near-far effect that occurs if weak radio signals from remote terminals have an increased probability of being lost in excessive noise or interference [7]-[12]. The analysis of channels with propagation delays is complicated by the fact that packet arrivals at the receiver do not exactly comply to an on-off switched Poisson process [9], as was considered in [2]. As the leading edge of the busy signal propagates, more and more terminals will be inhibited from potential transmissions, and the packet arrival rate at the base station will decrease gradually, rather than abruptly. Similarly, as soon as the channel is released by a transition from broadcasting a busy signal to an idle signal, the arrival rate of packets will increase gradually.

The organization of the paper is as follows. In Section II, we present our traffic model and recapitulate a theoretical result on nonstationary Poisson processes. In Section III, we describe our system model and introduce the random variables of interest. In Section IV, we use the system and traffic model to find a description of the nonstationary arrival processes of packets at the base station. In Section $V$, we assess $I(r)$, the average duration of the idle period, as perceived by a terminal at a distance $r$ away from the base station. In Section VI, we assess $B(r)$, the average busy period duration as seen by a remote terminal. From there we make approximations by assuming light traffic, not because an exact analysis would be unfeasible, but since it simplifies analysis and thereby clarifies the presentation. In Section VII, the previously assessed quantities are used to calculate $Q(r)$, the probability of successful transmission of a packet by a terminal at distance $r$, which is given by:

$$
Q(r)=\frac{I(r)}{I(r)+B(r)} \cdot \operatorname{Pr}\{\text { no harmful interference }\}
$$

This equation expresses that a packet is received undamaged if it is transmitted in an idle period, so it will not collide with a packet that is being received already, and if it is not impaired by any incoming packets during its own reception. Thereafter $Q(r)$ is used to assess the throughput performance of the system using the well known formula

$$
S_{t}=\int_{0}^{R} G(r) Q(r) 2 \pi r d r
$$

where $G(r)$ is the offered traffic at distance $r$, with $0 \leq r \leq$ $R$, which is defined as the average number of transmission attempts per area unit per unit of time. In Section VIII, the exact analysis is outlined, which is shown to improve the previous results only marginally. Section IX contains some concluding remarks.

\section{TRAFFIC MODEL}

Spatial Distribution: Packet transmission attempts will be described with a stationary spatially-distributed Poisson arrival process [1], [11], [12], [14]-[16]. Terminals are assumed to be located in a circular area with radius $R$. An infinite population of terminals is considered. For simplicity, in this paper, the attempted packet traffic is considered to be of uniform intensity throughout a service area, although our approach is also valid for non-uniform traffic distributions. The duration of a packet's transmission time is considered to be constant and is chosen as the unit of time. The average number of attempts per unit of area per packet time (ppt), originating from a distance $r$, is $G(r)$, which in this paper is set to $G(r)=G_{t} /\left(\pi R^{2}\right)$, with $0<r<R$ and $G_{t}$ the total attempted traffic. Retransmissions of previously collided or inhibited packets are included in $G_{t}$.

Temporal Distribution: We address the steady-state performance of the network, so the attempt process is stationary, which is equivalent to the independence of time of $G(r)$. Nonetheless, in the next section it will be shown that our system model gives rise to a time dependent packet arrival rate at the base station, because of the transmission inhibit signal that does not reach all terminals simultaneously. In other words, packets arrive at the base station, according to a non-stationary Poisson arrival process.

We recapitulate the generalization of a Poisson arrival process to a time-dependent, or nonstationary Poisson arrival process. Let $N(t, t+\Delta t)$ denote the number of arrivals in $[t, t+\Delta t)$. Suppose that for a positive, bounded function $\lambda(t)$, as $\Delta t \rightarrow 0$,

1. $\operatorname{Pr}\{N(t+\Delta t)=0\}=1-\lambda(t) \Delta t+o(\Delta t)$

2. $\operatorname{Pr}\{N(t+\Delta t)=1\}=\lambda(t) \Delta t+o(\Delta t)$

3. $\operatorname{Pr}\{N(t+\Delta t)>1\}=o(\Delta t)$.

The distribution of the number of arrivals in a time interval of $[t, t+T$ ), if $T>0$ is then given by (cf. [17], pp. 172,340)

$$
\operatorname{Pr}\{N(t, t+T)=n\}=\frac{(\bar{\lambda} T)^{n}}{n !} \exp (-\bar{\lambda} T)
$$

where $\bar{\lambda}$ is defined as

$$
\bar{\lambda} \triangleq \frac{1}{T} \int_{t}^{t+T} \lambda(t) d t
$$

i.e., as the arrival rate averaged over the interval $[t, t+T)$.

\section{SYSTEM MODEL}

A terminal at a distance $r$ from the central station, cycles through the following three time intervals, which are defined in the order that they transpire:

The IDLE period, with duration $i(r)$, is the interval of time during which the following two conditions are satisfied simultaneously: All terminals at distance $r$ receiving an idle signal and a packet transmitted by any of these terminals, would arrive at an idle receiver.

Because of propagation delays, the duration $i(r)$ of the idle period is a function of the location of the terminal.

The VULNERABLE period, with duration $v(r)$, is the interval of time during which any terminal at distance $r$ still receives an idle signal, but if it would transmit a packet in this interval, the packet would arrive at an already busy receiver. That is a receiver that is in the process of receiving another packet. 


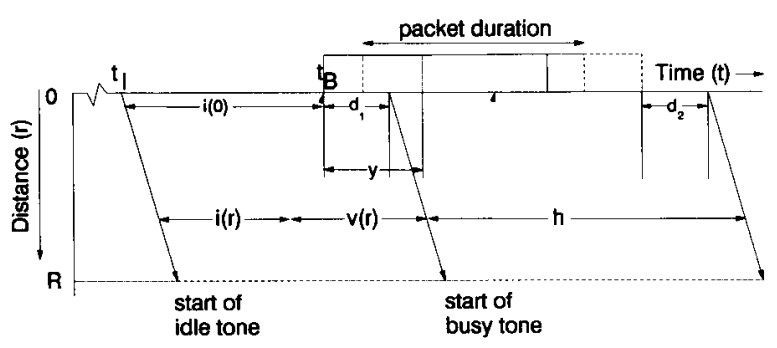

Fig. 1. Time-space diagram for inbound (. . .) and outbound (-) signals traveling over the radio channel.

This period too varies with the distance between the central station. Finally, we define the period that concludes a full cycle.

The INHIBITED period, with duration $h$, is the period during which a busy signal is received by a terminal. A vulnerable and subsequent inhibited period together is called a BUSY period $b(r)=v(r)+h$ (see also [2]).

The total duration of a cycle, or the period from beginning of a inhibited period until the beginning of the next inhibited period is $a=i(r)+b(r)$. Since packet arrivals are random, $a, h, i(r), b(r)$ and $v(r)$ are stochastic variables. Corresponding expectation values are denoted in capitals: as $A, H, I(r), B(r)$ and $V(r)$, respectively.

Fig. 1 illustrates the definitions above. It also shows that $a$ and $h$ are independent of the distance $r$. In the analysis of channels without propagation delays, these periods can be drawn on a (one-dimensional) time axis, as is done in [2]. However, to assess the effect of propagation delays, the distance to the base station is taken as a second dimension in the 'time-space' diagram of Fig. 1. A packet transmitted in the idle period arrives at an idle receiver in the central station. During a cycle, the first packet arriving at the idle receiver is called the 'initiating' packet. After a processing delay of duration $d_{1}$ time units, the central station starts transmitting a busy signal. This busy status is maintained for the entire duration of the packet reception. It is possible that during the reception of the initiating packet, a number of other, so called 'interfering' packets arrive, since the busy signal was not yet received by the originating terminals. The busy signal is being broadcast as long as overlapping packets are being received by the central station. When the reception of the last of the colliding packets terminates, the idle signal is transmitted after a delay $d_{2}$. The processing delays $d_{1}$ and $d_{2}$ are parameters of the central station and are taken to be fixed. Impairments of the feedback channel, other than propagation delays, such as noise or bandwidth limitations [4], are not incorporated in our analysis.

We define time $t_{I}$ as the start of the cycle under study, when the base station starts broadcasting an idle signal. This signal arrives at a distance $r$ from the base station at the instant $t_{I}+r / c$, with $c$ the speed of light normalized in respect to the packet transmission time. A terminal has permission to transmit only while receiving an idle signal. Thus, the first instant when a packet from a distance $r$ can possibly arrive at the base station is $t_{I}+2 r / c$. The actual moment of arrival of the initiating packet at the central station, which is the beginning of a busy period at $r=0$, is denoted as $t_{B}$, where $t_{B}=t_{I}+i(0)$. For a terminal at distance $r>0$, however, the idle period already ends at $t_{B}-r / c$. Taking into account the possibility that a terminal starts transmitting before the idle signal has reached all terminals, which means that $i(0)<2 R / c$, the idle period at a distance $r$ is found as the interval

$$
\left[t_{I}+\frac{r}{c}, t_{I}+\max \left\{\frac{r}{c}, i(0)-\frac{r}{c}\right\}\right)
$$

which makes the duration of the idle period $i(r)=$ $\max \{0, i(0)-2 r / c\}$.

After reception of the initiating packet, the central station starts broadcasting the busy signal with a delay $d_{1}$. The vulnerable period at a distance $r$ is found as the time interval

$$
\left[t_{I}+\max \left\{\frac{r}{c}, i(0)-\frac{r}{c}\right\}, t_{I}+i(0)+d_{1}+\frac{r}{c}\right)
$$

with duration $v(r)=d_{1}+\min (2 r / c, i(0))$. Colliding packets may be transmitted from a distance $r$ until the busy signal arrives at $t_{B}+d_{1}+2 r / c$. The inhibited period terminates $1+d_{2}$ units of time after the arrival of the last packet in the cycle is considered. In Fig. 1, we introduce the auxiliary random variable $Y$, that represents the interval between the time of arrival of the initial packet and the last of the interfering packets in the current cycle. Therefore the inhibited period is the interval

$$
\left[t_{B}+d_{1}+\frac{r}{c}, t_{B}+Y+1+d_{2}+\frac{r}{c}\right) .
$$

As noted before, $h$ is independent of $r$. We will assume in this paper that $d_{1}+t_{p}<1$, where $t_{p}=2 R / c$ is defined as the round-trip delay, which guarantees that interfering packets, sent because the originating terminal did not receive the busy tone yet, is bound to collide with the initiating packet in the current cycle, so it will not arrive some time in the next cycle.

\section{PaCket ARrival Rate at the Base Station}

At the base station, between $t_{I}$ and $t_{I}+t_{p}$, the packet arrival rate steadily grows from 0 to $G_{t}$ ultimately, as more and more terminals receive the idle tone, and are allowed to start transmitting. After the reception of the initiating packet at $t_{B}$, the arrival rate keeps increasing, if not at its maximum 


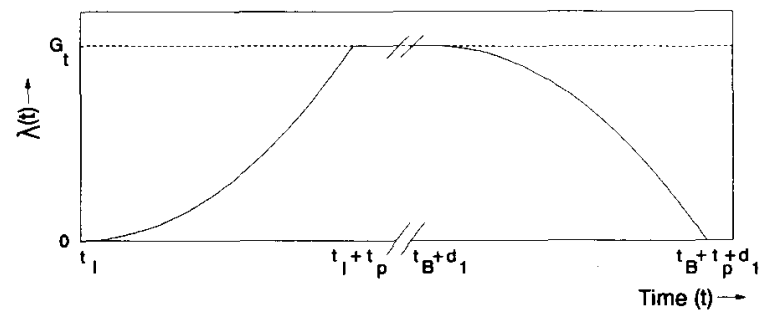

Fig. 2. The rate of arrival $\lambda(t)$ versus time after the start of an idle period $\left(t_{I}\right)$ until the eventual arrival of the inhibit signal at the boundary of the service area at $t_{I}+d_{1}+t_{p}$, for $G_{t}=1 \mathrm{ppt}$

already, until the processing time $d_{1}$ has expired, and then starts falling off to become zero, as the propagating busy tone eventually reaches the boundary of the circular service area. The expression for the arrival rate during the cycle under study is

$$
\left\{\begin{array}{l}
\int_{0}^{\min \left\{\mathrm{R}, \mathrm{c}\left(\mathrm{t}-\mathrm{t}_{\mathrm{I}}\right) / 2\right\}} G(r) 2 \pi r d r \\
\text { for } 0 \leq y<t_{B}+d_{1} \\
\int_{\min \left\{\mathrm{R}, \mathrm{c}\left(\mathrm{t}-\mathrm{t}-\mathrm{t}_{1}\right) / 2\right\}}^{\left.\left.\min -\mathrm{t}_{\mathrm{B}}-\mathrm{d}_{1}\right) / 2\right\}} G(r) 2 \pi r d r \\
\quad \text { for } t_{B}+d_{1} \leq t<t_{B}+Y+1+d_{2}
\end{array}\right.
$$

Note that while the arrival rate may not always be able to reach its peak value $G_{t}$, it will always drop to zero before the end of the busy cycle, since we assumed $d_{1}+t_{p}<1$, which guarantees that all packets that were sent by terminals before they were silenced by the busy signal, i.e. before $t_{B}+$ $d_{1}+{ }^{r} / c$, overlap with the initiating packet. For convenience, we introduce $\lambda_{I}(t)=\lambda\left(t-t_{I}\right)$ and $\lambda_{B}(t)=\lambda\left(t-t_{B}\right)$. The packet arrival rate as a function of time for our choice of $G(r)$ is depicted in Fig. 2 for the case where it does reach its peak $G_{t}$.

\section{DuRation OF THE IDLE PerioD}

In order to establish the idle period pdf, we use (3), to find that the probability that no packet arrives at the central receiver during the period $\left(t_{I}, t_{I}+x\right)$ is

$$
\operatorname{Pr}\left\{N\left(t_{I}, t_{I}+x\right)=0\right\}=\exp \left(-\int_{0}^{x} \lambda_{I}(t) d t\right) .
$$

Note that $t_{B}>x$, if no arrivals may occur in $[0, x]$, and that $\lambda_{I}(t)=\min \left\{G_{t}, G_{t} t^{2} / t_{p}^{2}\right\}$ for $t \leq x<t_{B}$, which follows from (8). The cumulative distribution function (cdf) $F_{i(0)}(x)$ of the lapse of idle time $i(0)$ at the central station $(r=0)$ then becomes

$$
\begin{aligned}
F_{i(0)}(x) & \triangleq \operatorname{Pr}\{i(0)<x\}=1-\operatorname{Pr}\left\{N\left(t_{I}, t_{I}+x\right)=0\right\} \\
& =1-\exp \left\{-\int_{0}^{x} \lambda_{I}(t) d t\right\} .
\end{aligned}
$$

It is seen that, in contrast to the conventional case with stationary Poisson arrivals $(\lambda(t)$ is constant), the idle time is not exponentially distributed. The average duration $I(r)$ of an idle period is obtained as

$$
\begin{aligned}
I(r) \triangleq & E\left[\max \left(0, i(0)-\frac{2 r}{c}\right)\right] \\
= & \int_{\frac{2 r}{c}}^{\infty}\left(x-\frac{2 r}{c}\right) \frac{d F_{i(0)}(x)}{d x} d x \\
= & \int_{\frac{2 r}{c}}^{t_{p}}\left(x-\frac{2 r}{c}\right) \lambda_{I}(x) \exp \left\{-\int_{0}^{x} \lambda_{I}(t) d t\right\} d x \\
& +\int_{t_{p}}^{\infty}\left(x-\frac{2 r}{c}\right) \lambda_{I}(x) \\
& \cdot \exp \left\{-\int_{0}^{t_{p}} \lambda_{I}(t) d t-\int_{t_{p}}^{x} \lambda_{I}(t) d t\right\} d x .
\end{aligned}
$$

The first term is evaluated using integration by parts, yielding

$$
\begin{aligned}
& -\int_{\frac{2 r}{c}}^{t_{p}}\left(x-\frac{2 r}{c}\right) d \exp \left\{-G_{t} \int_{0}^{x} \frac{t^{2}}{t_{p}^{2}} d t\right\} \\
& =-\left(t_{p}-\frac{2 r}{c}\right) \exp \left\{-G_{t} \frac{t_{p}}{3}\right\} \\
& +\int_{\frac{2 x}{c}}^{t_{p}} \exp \left\{-G_{t} \frac{x^{3}}{3 t_{p}^{2}}\right\} d x .
\end{aligned}
$$

The second term becomes

$$
\begin{aligned}
& \int_{t_{p}}^{\infty}\left(x-\frac{2 r}{c}\right) G_{t} \exp \left\{-G_{t}\left(\frac{t_{p}}{3}+x-t_{p}\right)\right\} d x \\
& =\left(t_{p}-\frac{2 r}{c}+\frac{1}{G_{t}}\right) \exp \left\{-\frac{1}{3} G_{t} t_{p}\right\} .
\end{aligned}
$$

Adding both terms we find

$$
\begin{aligned}
I(r)= & \int_{\frac{2 r}{c}}^{t_{p}} \exp \left\{-G_{t} \frac{x^{3}}{3 t_{p}^{2}}\right\} d x+\frac{1}{G_{t}} \exp \left\{-\frac{1}{3} G_{t} t_{p}\right\} \\
= & \frac{1}{3} \sqrt[3]{\frac{3 t_{p}^{2}}{G_{t}}}\left[\gamma\left(\frac{1}{3}, \frac{1}{3} G_{t} t_{p}\right)-\gamma\left(\frac{1}{3}, \frac{2}{3} G_{t} \frac{r^{3}}{R^{2} c}\right)\right] \\
& +\frac{1}{G_{t}} \exp \left\{-\frac{1}{3} G_{t} t_{p}\right\}
\end{aligned}
$$

where the incomplete gamma function is defined as

$$
\gamma(\alpha, x) \triangleq \int_{0}^{x} e^{-t} t^{\alpha-1} d t
$$




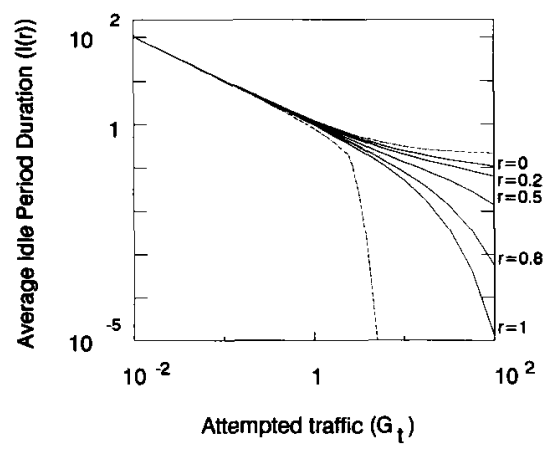

Fig. 3. Average duration of the idle period $I(r)$ (一) versus attempted traffic load $G_{t}$ for various distances. Maximum round-trip delay $t_{p}=0.2$. Bounds $G_{t}^{-1}+t_{p}, G_{t}^{-1}-t_{p}(-)$ and $G_{t}^{-1}(\ldots)$.

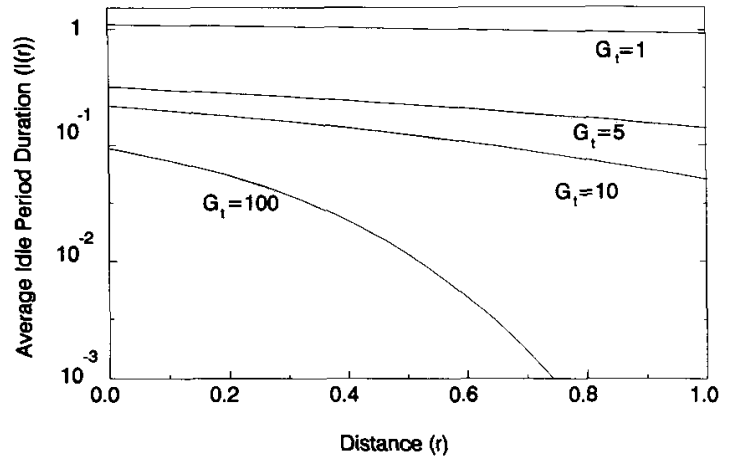

Fig. 4. Average duration of the idle period $I(\tau)(-)$ versus distance $r$ for various traffic loads, and linear approximation $\max (0 . I(0)-2 r / c)(\cdots)$ Maximum round trip delay $t_{p}=0.2$.

For channels without propagation delays $\left(t_{p}=0\right)$, (9) confirms that the average duration of the idle period becomes $I(r)=G_{t}^{-1}$. Fig. 3 presents $I(r)$ as a function of $G_{t}$ for different $r$. Also shown as dotted lines are the bounds $G_{t}^{-1} \leq I(0) \leq G_{t}^{-1}+t_{p}$ and $G_{t}^{-1}-t_{p} \leq I(R) \leq G_{t}^{-1}$. It is seen that for a terminal at a specific distance, the average duration of the idle period rapidly reduces to zero, if the attempted traffic load $G_{t}$ exceeds a certain intensity. Fig. 4 shows $I(r)$ as a function of $r$, and the corresponding linear approximations $I(r) \approx \max (0, I(0)-2 r / c)$ for small $G_{t}$

\section{DuRation of THE Busy Period}

Compared to the investigation of $I(r)$, the analysis of the average duration of the vulnerable and inhibited periods is relatively complicated. This is caused by the fact that not all terminals have received the idle tone at the moment that the initiating packet is sent, which occurs if the foregoing idle period $i(0)$ is shorter than the round-trip delay $t_{p}$. In that case, the duration of the busy period comes to depend on the idle period duration. From here on we simplify the analysis by making an assumption which is particularly valid, if the traffic load is light. In the Section VIII, we will come back to the exact approach, which is not impractical, but more tedious.

Our simplifying assumption states that $i(0) \geq t_{p}$, which is reasonable if $F_{i(0)}\left(t_{p}\right)=1-\exp \left\{\frac{-1}{3} G_{t} t_{p}\right\} \approx \frac{1}{3} G_{t} t_{p}$ is negligibly small. In that case, each idle period has nonzero duration $i(r)=i(0)-2 r / c$ for every location in the service area. We conclude from equation (5), that under our assumption, the arrival rate after $t_{B}$, becomes independent of $i(0)=t_{B}-t_{I}$, since $\left(t_{B}-t_{I}\right) c / 2 \geq R$, so that the upper bounds of both integrals reduce to $R$. We note that in order to find $A$, being independent of $r$, we only need to obtain $H$, as $A=I(0)+B(0)=I(0)+V(0)+H=I(0)+d_{1}+H$.

The time gap $y$ between the arrival of the initiating packet and the last interfering packet in the vulnerable period has the cdf

$$
\begin{aligned}
& F_{y}(x) \triangleq \operatorname{Pr}\{y \leq x\} \\
& = \begin{cases}0 & \text { if } x<0\end{cases} \\
& \left\{\operatorname{Pr}\left\{\text { no arrivals in }\left[t_{B}+x, t_{B}+d_{1}+t_{p}\right)\right\} \quad \text { if } x>0\right. \\
& = \begin{cases}0 & \text { if } x<0 \\
\exp \left(-\int_{x}^{d_{t}+t_{p}} \lambda_{B}(t) d t\right) & \text { if } 0<x<d_{1}+t_{p} \\
1 & \text { if } x>d_{1}+t_{p} .\end{cases}
\end{aligned}
$$

Inserting (5) gives

$$
F_{y}(x)= \begin{cases}0 \quad & \text { if } x<0 \\ \exp \left(-\frac{2}{3} t_{p} G_{t}-d_{1} G_{t}+x G_{t}\right) & \text { if } 0<x<d_{1} \\ \exp \left(-G_{t}\left[t_{p}+d_{1}-y\right]+\frac{1}{3} G_{t}\left[t_{p}-\frac{\left(y-d_{1}\right)^{3}}{t_{p}^{2}}\right]\right) & \quad \text { if } d_{1}<x<d_{1}+t_{p} \\ 1 \quad & \text { if } x>d_{1}+t_{p} .\end{cases}
$$

At $y=0$, this cdf exhibits a step of a size equal to the probability that no interfering packet is present. The average duration of the inhibited period is $H=Y+1-d_{1}+d_{2}$, or

$$
\begin{aligned}
H= & \left.y F_{y}(y)\right|_{0} ^{d_{1}+t_{p}}-\int_{0}^{d_{1}+t_{p}} F_{Y}(y) d y+1-d_{1}+d_{2} \\
= & t_{p}-\int_{0}^{d_{1}} \exp \left\{\left(-\frac{2}{3} t_{p}-d_{1}+y\right) G_{t}\right\} d y-\int_{d_{1}}^{d_{1}+t_{p}} \\
& \cdot \exp \left\{-G_{t}\left(\frac{2}{3} t_{p}+d_{1}-y+\frac{\left(y-d_{1}\right)^{3}}{t_{p}^{2}}\right)\right\} d y+1+d_{2} \\
= & t_{p}+e^{-\frac{2}{3} t_{p} G_{t}}\left[-\frac{1}{G_{t}}\left(1-e^{-d_{1} G_{t}}\right)\right. \\
& \left.-\int_{0}^{t_{p}} \exp \left\{x G_{t}-\frac{x^{3}}{t_{p}^{2}} G_{t}\right\} d x\right]+1+d_{2}
\end{aligned}
$$

Limiting cases are $H \rightarrow 1-d_{1}+d_{2}$ for $G_{t} \rightarrow 0$ and $H \rightarrow$ $1+t_{p}+d_{2}$ for $G_{t} \rightarrow \infty$. For channels without propagation delays $\left(t_{p} \rightarrow 0\right), H \rightarrow\left(1-\exp \left\{-d_{1} G_{t}\right\}\right) G_{t}^{-1}+1+d_{2}$, which is in agreement with [2]. Fig. 5 portrays equation (12) as well as the exact solution, to be discussed in Section VIII, for comparison.

\section{ThroughPUt PERformanCE ANALYSIS WITH RECEIVER CAPTURE}

In a realistic radio channel, an initiating packet may be received correctly despite the presence of weak interfering pack- 


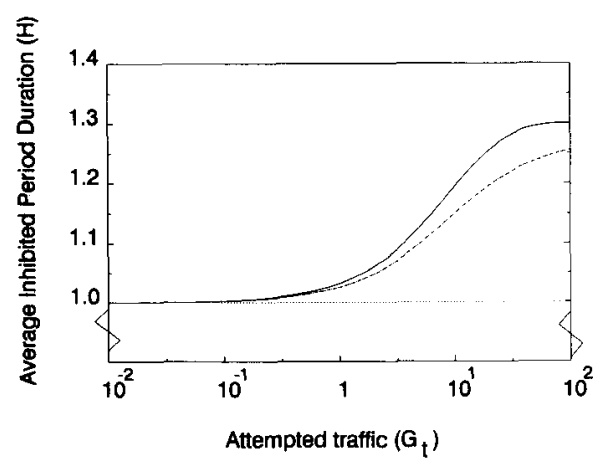

Fig. 5. Average duration of the inhibited period $H$ versus attempted traffic load $G_{t}$, according to approximate (-) and exact method (-). Bounds $1-d_{1}+d_{2}(---)$ and $1+d_{2}+t_{p}(\cdots)$. Maximum round trip delay $t_{p}=0.2$, processing delays $d_{1}=d_{2}=0.1$.

ets. This capture effect has been studied extensively for random access channels employing slotted ALOHA [1], [7]-[9], [14]-[16], [18]-[20], and for ISMA with fixed (distanceindependent) propagation delays [8], [9], [11], [12]. The vulnerability-circle model, presented in 1977 by Abramson [1], can conveniently be incorporated in the present analysis, while it is not within the scope of this paper to address more refined capture models, e.g., those including aspects of channel fading, modulation and coding.

In this model, an initiating packet from distance $r$ is assumed to be received correctly, if none of the interfering packets arrives from a distance closer than $c_{z} r$, with $c_{z}$ a system constant. Besides, we assume that the receiver never switches over to an interfering packet once it is synchronized to the initiating packet. By choosing $c_{z}=\infty$, the analysis reduces to that of a system without receiver capture. The probability of no harmfully interfering packets is now easily found by employing (3) after we have modified $\lambda(t)$ from equation (5), to exclude packets from outside the circle with radius $c_{z} r$. This is done by replacing $R$ with $\min \left(R, c_{z} r\right)$. The modified arrival rate $\lambda^{\prime}(t)$ becomes

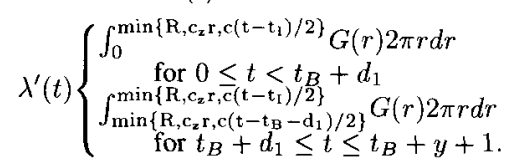

The probability of no harmful interference, given that the initiating packet was transmitted by a terminal at distance $r$, is found as

$$
\begin{aligned}
& \operatorname{Pr}\{\text { no harmful interference } \mid r\} \\
& =\exp \left\{\int_{0}^{d_{1}+t_{p}} \lambda^{\prime}\left(t-t_{B}\right) d t\right\} \\
& =\exp \left\{-\left(\frac{\min \left\{R, c_{z} r\right\}}{R}\right)^{2}\left[d_{1}+\frac{2}{3} t_{p}\right] G_{t}\right\} .
\end{aligned}
$$

Fig. 6 presents numerical results of $Q(r)$ for $G_{t}=2 \mathrm{ppt}$ We see that the probability of successful transmission tends

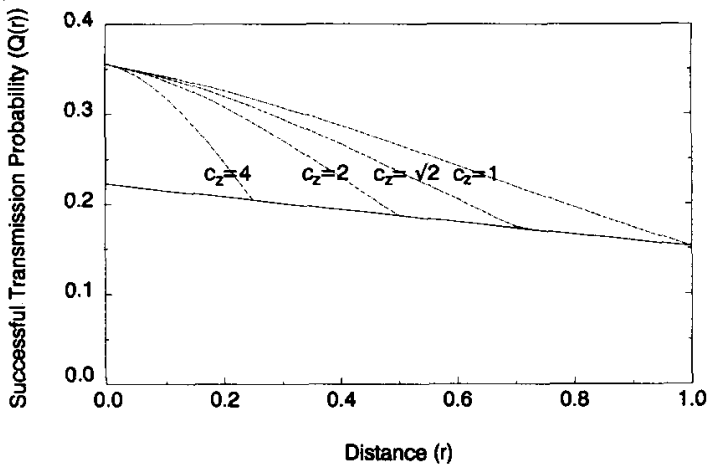

Fig. 6. The probability of successful transmission $Q(r)$ versus distance $r$ for $t_{p}=0.2$ and $d_{1}=d_{2}=0.1$ for various capture parameters. The total channel traffic load $G_{t}$ is 2 ppt.

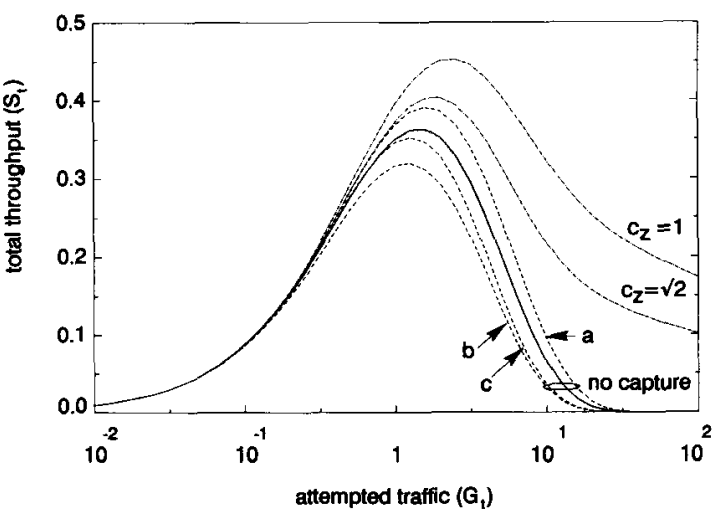

Fig. 7. Throughput $S_{\ell}$ versus attempted traffic $G_{t}$ for $d_{1}=0.1, d_{2}=0.1$, $t_{p}=0.2$ with capture (-.--) and without capture, approximate $(\cdot \cdots)$ and exact (-) technique [18]. Fixed delay for all terminals (-- ) (a): $d_{1}=0.2$, $d_{2}=0.2, t_{p}=0,(\mathrm{~b}): d_{1}=0.3, d_{2}=0.3, t_{p}=0$ and (c): $d_{1}=0.3$, $d_{2}=0.1, t_{p}=0$.

to decrease linearly with distance, particularly for larger $r$. The total channel throughput $S_{t}$, expressed in the average number of successfully received packets per unit of time, is obtained with equation (2) through numerical integration, and illustrated in Fig. 7. Also drawn are the lines produced by various approximations, to be described in the next paragraph, and an exact analysis, which will be discussed in the next section.

For CSMA, it was previously suggested [2] to approximate the effect of the variable propagation delay, by assuming all terminals to be at an identical (worst case) distance from the base station. This corresponds to introducing the modified delays $d_{1}^{\prime}=d_{1}+R / c$ and $d_{2}^{\prime}=d_{2}+R / c$ and taking the modified round trip delay $t_{p}^{\prime}=0$ in our model. For ISMA, three different approximations, designated $a, b$, and $c$, will be compared for the special case $d_{1}=0.1, d_{2}=0.1$ and $t_{p}=0.2$. Approximation $a$ adds the one-way propagation delay to both $d_{1}$ and $d_{2}\left(d_{1}^{\prime}=0.2, d_{2}^{\prime}=0.2\right)$. Approximation 


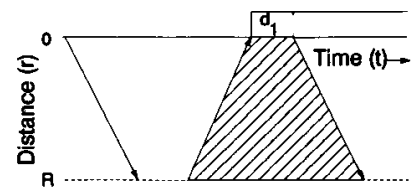

$\mathrm{i}(0)>\mathrm{t}_{\mathrm{p}}$

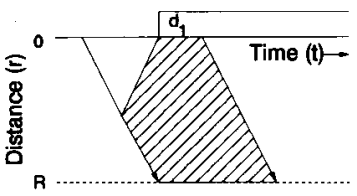

$\mathbf{i}(0)<\mathbf{t}_{\mathbf{p}}$

Fig. 8. Time-space diagram and vulnerable period $(/ / /)$ for the events $i(0)>t_{p}$ and $i(0)<t_{p}$.

$b$ adds the round-trip delay to both $d_{1}$ and $d_{2}\left(d_{1}^{\prime}=0.3, d_{2}^{\prime}=\right.$ $0.3)$. Approximation $c$ adds the round-trip delay to $d_{1}$, but no propagation delay is accounted for in $d_{2}\left(d_{1}^{\prime}=0.3, d_{2}^{\prime}=\right.$ 0.1 ).

Although approximation $a$ offers worst case results for the throughput of CSMA [2], it overestimates the throughput for ISMA. Approximation $b$, on the other hand, yields relatively conservative throughput estimates. Approximation $c$ gives about $5 \%$ lower results than the exact analysis, and may offer a sufficiently tight bound for practical system design, particularly in the range $G_{t}<1 \mathrm{ppt}$.

This conclusion is understood by recognizing that approximation $c$ takes account of the effect that the round trip delay substantially widens the vulnerable period, and thus increases the expected number of interfering signals. Approximation $a$ overestimates throughput, mainly because it tends to underestimate the number of interfering signals. As can be seen from (14), for a uniform distribution of the attempted traffic, the probability of no packets interfering with an initiating packet is $\exp \left\{-d_{1}-2 / 3 t_{p}\right\}$, thus $d_{1}$ is effectively prolonged by $133 \%$ of the one way propagation delay.

Finally, the effect of receiver capture $(z=1$ and $\sqrt{2})$ is seen to be significant, particularly for high offered traffic loads $\left(G_{t}>1\right)$.

\section{EXaCt ANALYSIS}

Fig. 8 illustrates the effect of the event $i(0)<t_{p}$ on timespace volume from where the traffic originates. This volume determines the length of the vulnerable period as well as the probability of no harmful interference. In that case, $H$ is obtained by first calculating $H(x)$, which is the average duration of the inhibited period for a given $i(0)=x$. Then we uncondition as in

$$
H=\int_{0}^{\infty} H(x) f_{i(0)}(x) d x
$$

The event $i(0)<t_{p}$ also affects the probability of no harmful interference. In a similar manner, we first obtain $\operatorname{Pr}\{$ no harmful interference $\mid r, i(0)\}$, which is the probability of harmful interference, given that the originating terminal was a distance $r$ away from the base station, and given that the actual duration of the idle period was $i(0)$. Then we uncondition as follows

$$
\begin{aligned}
\operatorname{Pr} & \{\text { no harmful interference } \mid r\} \\
& =\int_{0}^{\infty} \operatorname{Pr}\{\text { no harmful interference } \mid r, x\} f_{i(0)}(x) d x .
\end{aligned}
$$

Evaluation of the previous two integrals becomes tiresome because an analytic expression of the integrand only exists for sub-intervals over the integration variable, and various segments of the time space volume are to be distinguished in the integration [21]. The analysis presented in [21] is therefore not repeated here, but numerical results are given in Fig. 5 and Fig. 7 to discuss the accuracy of the approximate model in comparison with the exact average inhibited period duration and throughput. It is seen in Fig. 5 that the duration of $H$ is overestimated by (12). This leads to an underestimation of $Q(r)$ and $S_{t}$. Moreover, if $i(0)<t_{p}$, the time-space volume from which interfering packets may arrive is smaller than assumed in (14). Hence, the number of interfering signals is overestimated. This also leads to an underestimation of the channel performance. However, the effect of these approximations on throughput turns out to be negligible (see Fig. 7).

\section{Concluding Remarks}

Propagation delays cause unfairness in a random-access radio network employing inhibit sense multiple access. The principal contribution to this unfairness is caused by the effect that the idle period decreases almost linearly with the distance between the terminal and the central station. The unfairness caused by propagation delays is additional to the unfairness caused by the fact that weak signals from remote terminals experience a higher probability of being lost in a collision. It was seen that for reasonable traffic loads, say $G_{t}<5$, propagation delays cause less unfairness than propagation attenuation. At high traffic loads, delays are expected to become the major cause of unfair access probabilities for remote terminals: beyond a certain offered traffic load, terminals at a certain distance experience an highly limited probability to successfully transmit a data packet.

Exact analysis of ISMA with propagation delays is a tedious task. However, an approximate approach, taking in account of the duration of the idle period exactly, but ignoring the effect that overlapping cycles have on the duration of the busy period, proved to give relatively accurate results. Previously, the performance of random access in packet radio networks was assessed from a worst-case propagation delay. If this approximation is used, the maximum round-trip delay is best included in the delay $d_{1}$ occurring at the onset of the busy signal, rather than including the maximum one-way propagation time in both processing delays $d_{1}$ and $d_{2}$. In the former method, the effect that round-trip delays substantially widen 
the vulnerable period, and thus increase the expected number of interfering signals, is taken into account more accurately. The approximation by means of fixed (distance-independent) propagation delays was seen to yield too pessimistic results for the throughput.

Presently, the development of public networks for packet switched mobile data communication and wireless office automation systems tends to focus on wireless communication links over shorter and shorter ranges. This seems to advert interest from propagation delays in the net. Note, however, that these propagation delays are to be considered in relation to the packet duration. Since the bit rates considered for personal and mobile communications are increasing sharply, the effects studied here are considered of particular interest to the design of future random-access systems.

\section{REFERENCES}

[1] N. Abramson, "The throughput of packet broadcasting channels," IEEE Trans. Commun, vol. COM-25, pp. 117-128, Jan. 1977.

[2] L. Klcinrock and F. A. Tobagi, "Packet switching in radio channels: Part I-Carrier Sense Multiple Access modes and their throughputdelay characteristics," IEEE Trans. Commun., vol. COM-23, no. 12 , pp. $1400-1416$, Dec. 1975

[3] F. Tobagi and L. Kleinrock. "Packet switching in radio channels part II-The hidden terminal problem in carrier sense multiple access and the busy tone solution," IEEE Trans. Commun., vol. COM-23, no. 12, pp. 1417-1433, Dec. 1975

14] O. Andrisano, G. Grandi and C. Raffaelli, "Analytical model for busy channel multiple access (BCMA) for packet radio networks in a Iocal environment," IEEE Trans. Veh. Technol., vol. VT-39. no. 4. pp a local environment,"

[5] M. L. Molle, K. Sohraby and A. N. Venetsanopoulos, "Space-time model of asynchronous CSMA protocols for local area networks," IEEE $J$ Select Areas Commun., vol. SAC-5, no. 6, pp. 956-968, July 1987

[6] "Technical characteristics and test conditions for non-speech and combined analogue speech/non-speech radio equipment with internal or external antenna connector, intended for the transmission of data. for use in the land-mobile service" (DRAFT), European Telecommunicalions Standards Institule.

[7] 3. Krebs and T. Frecburg, "Method and apparatus for communicating J. Krebs and $T$. Frecburg, "Method and apparatus for communicating
variable length messages between a primary station and remote stations at a data communications system," US patent no. $4519068,1985$.

18] R. Prasad and J. C. Ambak, "Capacity analysis of non-persistent inhibit sense multiple access in channels with multipath fading and shadowing," in Proc. 1989 Workshop on Mobile and Cordless Telephone Communications, IEE, London, pp. 129-134, Sept. 1989.

[9] K. J. Zdunek, D. R. Ueci and J. L. Locicero, "Throughput of nonpersistent Inhibit Sense Multiple Access with capture." Electonics Letters, vol. 25, pp. 30-32. Jan. 1989.

[10] J. G. Gardiner and A. I. A. Jabbar, "Performance of CSMA protocols in fading mobile radio environments." in Proc. 5th IEE Int. Conj. on Mobile Radio and Personal Communications. Warwick, UK. pp. 10-14 Dec. 11-14.

[11] J. P. M. G. Linnartz, "Near-far effects in some random-access radio systems with fading," 1990 BLCON International Conference in New Trends in Communication, Control and Signal Processing. Ankari, Turkey, pp. 504-510, July 2-5, 1990.

112] J. P. M. G. Linnartz, R. Hekmat and R. J. Venema, "Near-far effects in land mobile random-access networks with narrow-band Rayleigh fading channels," IEEE Trans. Veh. Tech., vol. 41, no. 1, pp. 77-90. Feb. 1992.

[13] C. Milne, "Transient behaviour of the interrupted Poisson Process," Journal of the Royal Statistical Society: Series B (Methodological), vol. 44 , no. 3, pp. 398-405, 1982.

114) J. C. Ambak and W. van Blitterswijk, "Capacity of slotted-ALOHA in a Rayleigh fading channel." IEEE J. Select Areas Commun., vol. SAC-5. по. 2, pp. 261-269, Feb. 1987.
115] J. P. M. G. Linnartz, R. Prasad, and J. C. Ambak. "Spatial distribution of traftic in a cellular ALOHA network." Archiv für Elektronik und Ubertragungstechnik, vol. 42, no. 1, pp. 61-63, Jan.-Feb. 1988

[16] R. Pluymers, "Computer simulation of a mobile packet radio system," Electronics Letters, vol. 24, no. 6, pp. 316-317, Mar. 17, 1988.

[17] G. Grimmet and D. Stirzaker, Probability and Random Processes, Clarendon Press, Oxford, Great Britain, 1990

[18] I. M. I. Habbab, M. Kavehrad and C.-E. W. Sundberg, "ALOHA with capture over slow and fast fading radio channels with coding and diversity," IEEE Joumal Selected Areas Commun., vol. SAC-7, no. 1 pp. 79-88, Jan. 1989

[19] K. Zhang and K. Pahlavan, "A new approach for the analysis of the slotted ALOHA local radio networks," in Proc. International Conference on Commun. /CC, Atlanta, pp. 1231-1235, April 1990.

$[20 \mid$ C. Lau and C. Leung, "Capture models for mobile packet radio networks," in Proc. Intemational Conference on Commun. ICC, Atlanta, pp. 1226-1230, April 1990.

[2]] R. J. Venema, "Inhibit sense multiple access in systems with propagation delays." M.Sc.E.E. graduation thesis A333, University of Technology, The Netherlands, Sept. 18, 1990.

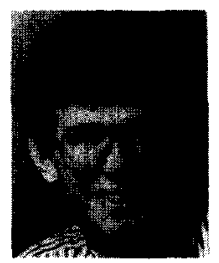

Jean-Paul M. G. Linnartz (S'85-M'87) received the M.Sc. E.E. degree in Electrical Engineering (Cum Laude) from Eindhoven University of Technology, The Netherlands, in 1986. During 1987-1988, he was with the Netherlands Organization for Applied Scientific Research, Physics and Electronics Laboratory F.E.L-T.N.O., The Hague, where he worked on UHF propagation Hague, where he worked was Assistant Professor at Delft University of was Assistant Professor at Delft University of
Technology, where he received a Ph.D. (Cum Laude) on multi-user mobile radio nets in December 1991. In June 1992, he received the "Veder Prize" (The Netherlands) for research on traffic analysis in mobile radio nets. He joined the Department of EECS at the University of Califormia at Berkeley as Assistant Professor in January 1992. In January 1993, he will publish his first book, entitled "Narrowband Land-Mobile Radio Networks,"

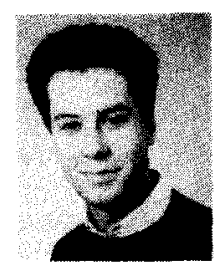

Geert A. Awater $(S 93)$ was born in Arnhem, The Netherlands in 1965 He received the degree from Twente University of Technology, in electri cal engineering in 1989 . He is currently working towards the Ph.D. degree at Delft University of Technology

In 1989, he joined the Telecommunications and Traffic Control Systems Group at the Department of Electrical Engineering, Delft University of Technology. He is currently engaged in research on nology. He is currently engaged in research on
teletraffic theory, with an emphasis on broadband switching networks and on broadband video communications.

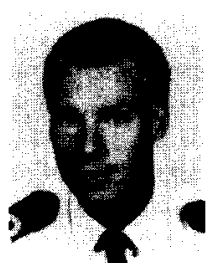

Robert-Jan Venema was born in Sao Paulo, Brazil in 1966. He received the M.Sc. degree in electrical engineering from Delft University of Technology, The Netherlands. He studied at the Royal Netherlands Naval College for three years.

In 1984, he joined the Royal Netherlands Navy. He sailed for a year and a half abroad a frigate as Officer of the Deck. After receiving his Master' degree, he completed his training as a Navy pilot at Groningen. 